\title{
Téoros
}

Revue de recherche en tourisme

\section{Un cadre d'évaluation du tourisme de nature durable en forêt publique québécoise}

\section{Véronique Desmarais et Louis Bélanger}

Volume 25, numéro 3, automne 2006

Tourisme et forêt, visions d'une gestion intégrée

URI : https://id.erudit.org/iderudit/1071014ar

DOI : https://doi.org/10.7202/1071014ar

Aller au sommaire du numéro

Éditeur(s)

Université du Québec à Montréal

ISSN

0712-8657 (imprimé)

1923-2705 (numérique)

Découvrir la revue

Citer cet article

Desmarais, V. \& Bélanger, L. (2006). Un cadre d'évaluation du tourisme de nature durable en forêt publique québécoise. Téoros, 25(3), 14-20.

https://doi.org/10.7202/1071014ar d'utilisation que vous pouvez consulter en ligne. 


\section{Un cadre d'évaluation du tourisme de nature durable en forêt publique québécoise}

\section{Véronique Desmarais et Louis Bélanger}

Dans les forêts publiques québécoises, l'enjeu du développement durable va bien au-delà de l'exploitation de la matière ligneuse et devrait s'appliquer à l'ensemble des utilisations de la forêt. C'est le cas du tourisme de nature durable. Le Québec, avec ses régions sauvages et ses forêts, a su faire sa marque dans le tourisme de nature. Qu'il s'agisse d'activités de plein air, d'aventure, d'observation ou de prélèvement faunique, le nature-based tourism, qui regroupe toutes les activités nécessitant un milieu naturel pour se concrétiser (Weaver, 2001), joue un rôle sans cesse grandissant dans la forêt publique québécoise. Le tourisme de nature durable, quant à lui, se définit comme

« toutes activités principalement axées sur l'observation ou l'appréciation de la nature, soit à des fins de découverte ou de pratique d'activités de plein air, et qui sont développées et gérées de façon à minimiser les impacts négatifs sur l'environnement et le milieu hôte et à maximiser les retombées économiques, notamment au plan local » (Tourisme Québec, 2003).

\section{Nécessité d'un cadre d'évaluation québécois}

La notion de tourisme de nature durable est incomplète au Québec et un cadre d'évaluation permettant d'établir le niveau de durabilité du tourisme reste à trouver. II faudrait élargir la réflexion portant sur le cadre de durabilité du tourisme de nature afin qu'elle aille au-delà de l'écotourisme et qu'elle couvre l'ensemble des activités de tourisme durable en milieu naturel (OMT, 2004). En ce qui concerne les forêts publiques québécoises exploitées par l'industrie forestière, l'application du tourisme durable est particulièrement complexe. II serait irréaliste de croire en un modèle d'application unique du tourisme de nature durable (Lück \& Kirstges, 2003) pour tous types de territoires forestiers publics du Québec. Que ce soit en raison des vocations du territoire, des contraintes du milieu ou des choix des gestionnaires touristiques, les types de tourisme de nature dite durable peuvent différer grandement, notamment en ce qui a trait aux activités offertes et à l'ampleur des infrastructures.

L'importance attribuée aux diverses vocations, elles-mêmes traduites sous forme de statuts de conservation, de droits octroyés et de permis d'occupation, permet d'établir les différents niveaux de tolérance par rapport aux impacts négatifs du tourisme sur le milieu et, donc, d'établir les types de tourisme qui y seront admis. Par exemple, le tourisme durable ne peut se conjuguer de la même façon dans un parc national dont la vocation première est la préservation de l'intégrité écologique et dans une réserve faunique ayant pour vocations l'exploitation forestière et la mise en valeur de la faune. Ainsi, même si le réseau des parcs nationaux présente un exemple d'initiative dans l'implantation d'un caractère durable à l'offre d'activités touristiques en milieu forestier, il ne peut servir de référence que dans les cas limités des sites où la préservation de l'intégrité écologique constitue la vocation première. II faudrait que cette réflexion faite pour les parcs nationaux depuis cinq ans en matière d'application du tourisme de nature durable soit aussi faite pour l'ensemble des forêts publiques québécoises, mais en prenant en considération les besoins propres à chacune. Cette réflexion reste à faire pour les territoires fauniques structurés, notamment, d'autant plus qu'ils jouent un rôle considérable en matière de tourisme de nature au Québec.
Ni les pourvoiries, ni les zones d'exploitation contrôlée (ZEC), ni le réseau des réserves fauniques de la SÉPAQ ne possèdent encore de cadre d'évaluation du tourisme de nature durable. Pourtant, ces territoires fauniques structurés représentent plus de $20 \%$ de l'ensemble des forêts publiques du Québec sous contrat d'approvisionnement et d'aménagement forestier (CAAF) (MRNF, 2006). Le cadre d'évaluation du tourisme de nature durable que nous suggérons s'applique donc aux territoires à vocations multiples ayant à la fois une vocation forestière et une vocation récréotouristique reconnues, qu'ils soient gérés par un gestionnaire unique, comme la forêt Montmorency, ou par des gestionnaires multiples, comme dans le cas des territoires fauniques structurés.

Aussi, il est à noter que les produits de tourisme de nature visés par le présent cadre d'évaluation se limitent aux activités non motorisées ou aux activités qui font l'utilisation de motorisés à des fins utilitaires seulement. Étant donné la complexité de la problématique liée aux activités touristiques de randonnée motorisée, telles le véhicule tout-terrain (VTT) et la motoneige, et les débats actuels soulevés en la matière, comme en témoignent les nombreux mémoires présentés lors de la consultation publique sur les véhicules hors route de Transports Québec en 2005, il serait préférable d'évaluer le tourisme de randonnée motorisée dans un cadre qui lui serait propre.

Bien qu'en terres publiques québécoises le tourisme de nature soit maintenant reconnu comme faisant partie de l'aménagement intégré des ressources, il n'y a aucune politique officielle de développement durable. Aussi, bien que Tourisme Québec reconnaisse, dans son plan d'action 2003-2008, la nécessité de se doter d'un cadre de référence de tourisme de nature durable, il n'existe pas 
encore de modèle québécois applicable qui permette d'encadrer ce type d'exploitation des ressources et de tendre davantage vers la conservation des milieux.

\section{Le double défi lié à l'exploitation forestière}

L'implantation du tourisme durable représente un défi d'autant plus difficile pour l'industrie touristique en milieu forestier public que celle-ci se voit confrontée à l'exploitation forestière sur la plus grande partie de la forêt. Cela constitue donc un défi supplémentaire pour le secteur du tourisme de nature, puisqu'il faut désormais, pour être durable, assurer la durabilité du produit touristique, mais aussi la durabilité du milieu d'accueil. Les divergences quant aux intérêts et aux besoins propres aux industries touristique et forestière font de leur harmonisation une importante problématique (Bélanger, 1998) pouvant rendre précaire la viabilité des entreprises touristiques. Ne peut être durable qu'un produit touristique offert là où la qualité du milieu est assurée. Si ces deux utilisateurs doivent se partager un même territoire et qu'aucune entente officielle n'est établie entre eux, des critères tels la tranquillité et l'esthétisme paysager ne pourront être garantis pendant et après les opérations forestières et, ainsi, l'offre touristique ne pourra pas être qualifiée de durable.

\section{Cadre d'évaluation du tourisme de nature durable en forêt}

Comme la conservation et la mise en valeur des paysages, des ressources naturelles et des richesses culturelles peuvent être qualifiées d'essentielles au sein de l'offre touristique en forêt, le Québec se doit de mettre en œuvre les moyens nécessaires pour implanter le tourisme de nature durable sur son territoire. Pour ce faire, nous proposons un cadre d'évaluation adapté aux réalités des forêts publiques québécoises, où les industries forestière et touristique se partagent un même territoire et visent à évaluer le niveau de durabilité des organisations touristiques au regard de leur gestion environnementale, sociale et économique. Ce modèle, jusqu'à aujourd'hui inexistant au Québec, est issu des résultats du travail de maîtrise de Véronique Desmarais, sous la direction de Louis Bélanger. II se veut une contribution aux efforts d'encadrement du tourisme de nature durable initiés par

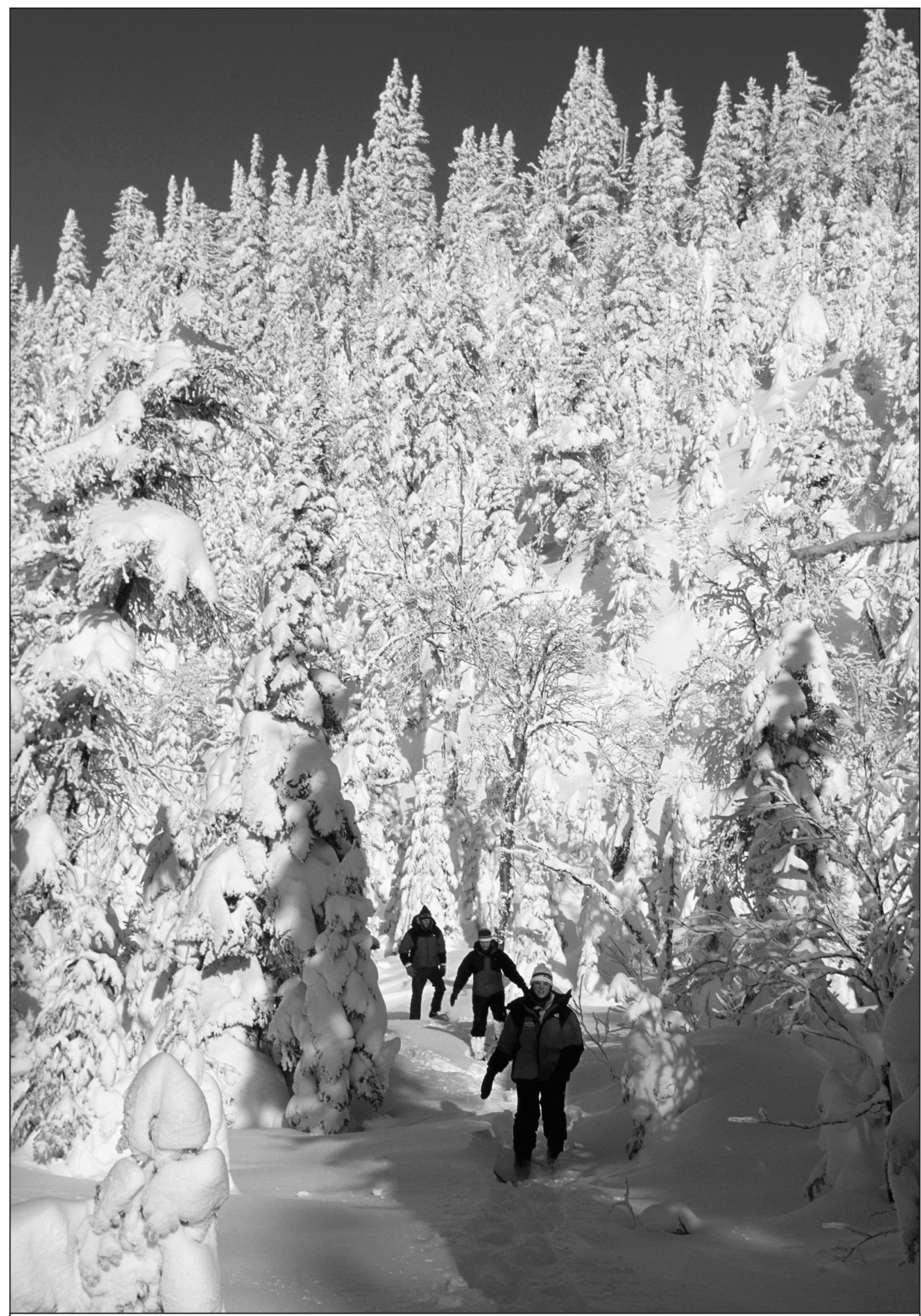

Raquette au parc national des Monts-Valin (Québec).

Photo : Jean-Pierre Huard/Tourisme Québec

Tourisme Québec, comme l'ont été les critères et les indicateurs du Conseil canadien des ministres des forêts pour l'aménagement forestier durable (CCMF, 2003).

Notre cadre couvre trois volets, soit les critères, les éléments et les indicateurs de performance. Les critères représentent les valeurs de durabilité que nous jugeons primordiales au sein de l'industrie québé- coise de tourisme de nature, tandis que les éléments servent à spécifier quels objectifs doivent ainsi être atteints. Les indicateurs, quant à eux, définissent de façon mesurable la performance des organisations dans l'application des critères de durabilité et permettent d'en évaluer la progression dans le temps. Cet article traite essentiellement des critères et des éléments constitutifs du cadre d'évaluation de tourisme de 
nature durable que nous suggérons. Les indicateurs de performance ne sont pas abordés, puisqu'ils varient selon les caractéristiques propres à chaque organisation touristique.

Notre cadre d'évaluation est établi de façon à couvrir l'ensemble des principes du tourisme durable, c'est-à-dire les nécessités environnementales de conservation de la biodiversité et de l'authenticité écologique, les impératifs sociaux du milieu et la viabilité économique de l'organisation touristique. Ils sont donc inspirés des principes internationaux de tourisme durable, particulièrement des recommandations du World Wildlife Fund (WWF, 2001), de l'Organisation mondiale du tourisme (OMT) pour la certification du tourisme durable (2003), du World Travel and Tourism Council (WTTC) et des recommandations de la Déclaration de Québec sur l'écotourisme (Ville de Québec, 2002). Ils s'appuient en outre sur des valeurs de durabilité présentes dans les chartes de tourisme durable, notamment celles de la Conférence mondiale du Tourisme durable tenue en Espagne en 1995 et de la Charte européenne du tourisme durable dans les espaces protégés (FPNRF, 1998), de même que sur les principes directeurs émis par Tourisme Québec dans son plan d'action 2003-2008.

\section{Critère 1 : Conformité à la notion de tourisme de nature}

L'organisation qui désire offrir du tourisme de nature durable devrait tout d'abord s'assurer que l'environnement dans lequel elle $œ u v r e$ est considéré comme naturel. La notion de milieu naturel correspond

à un milieu dans lequel la biodiversité et les processus écologiques n'ont pas été altérés de manière permanente ou à long terme par les activités humaines, qui maintient sa capacité de se régénérer et où la présence humaine ne modifie pas le paysage de manière importante ni ne le domine (Tourisme Québec 2003).

En ce sens, l'organisation devrait s'assurer que la proportion d'altération liée à l'aménagement des infrastructures et du site est telle qu'elle maintient un écosystème forestier et une superficie naturelle suffisants pour que le site soit qualifié de milieu naturel. De plus elle doit tenir compte que certaines activités, bien que liées à l'environ- nement, ne peuvent être considérées comme conformes au tourisme de nature. II s'agit en fait d'activités nécessitant un milieu naturel considérablement modifié, comme les terrains de golf et de ski alpin, ou se concrétisant dans un milieu non naturel, comme le jardin zoologique (Tourisme Québec, 2003).

\section{Tableau 1}

Cadre d'évaluation du tourisme de nature durable pour les forêts publiques aménagées

\begin{tabular}{|c|c|}
\hline \multicolumn{2}{|c|}{ Cadre d'évaluation du tourisme de nature durable pour les forêts publiques aménagées } \\
\hline & Éléments \\
\hline Conformité à la notion de tourisme de nature & $\begin{array}{l}\text { - Milieu naturel } \\
\text { - Activités de tourisme de nature }\end{array}$ \\
\hline Produit touristique de qualité & $\begin{array}{l}\text { - Plans, politiques et programmes de durabilité } \\
\text { - Qualité des activités et des services } \\
\text { - Sécurité }\end{array}$ \\
\hline Harmonisation des usages du territoire & $\begin{array}{l}\text { - Esthétisme et qualité des paysages } \\
\text { - Tranquillité des sites de récréation } \\
\text { - Sécurité routière }\end{array}$ \\
\hline Conservation des écosystèmes & $\begin{array}{l}\text { - Minimisation des impacts de ses propres infrastructures et } \\
\text { activités sur les espèces et les habitats } \\
\text { - Protection des espèces menacées et vulnérables et des } \\
\text { écosystèmes à haute valeur de conservation } \\
\text { - Observation et conservation de la faune } \\
\text { - Chasse, pêche et conservation de la faune }\end{array}$ \\
\hline Gestion de la pollution & $\begin{array}{l}\text { - Émission réduite et gestion durable des déchets / Recyclage } \\
\text { - Gestion durable des eaux résiduelles et de la consommation } \\
\text { d'eau potable } \\
\text { - Gestion durable de la consommation d'énergie dans les } \\
\text { bâtiments } \\
\text { - Gestion durable de la consommation de matériel } \\
\text { - Utilisation de moyens de transport à moindres impacts }\end{array}$ \\
\hline $\begin{array}{l}\text { Conservation et mise en valeur } \\
\text { du patrimoine culturel et historique }\end{array}$ & $\begin{array}{l}\text { - Protection du patrimoine culturel } \\
\text { - Respect de l'authenticité des cultures autochtones et des } \\
\text { communautés locales } \\
\text { - Respect des droits ancestraux et des droits découlant de } \\
\text { traités }\end{array}$ \\
\hline $\begin{array}{l}\text { Concertation, sensibilisation et information } \\
\text { sociales }\end{array}$ & $\begin{array}{l}\text { - Information et concertation dans la prise de décision et les } \\
\text { modes de gestion } \\
\text { - Formation des employés en matière de durabilité et de sécurité } \\
\text { - Programmes et activités de sensibilisation, d'éducation et } \\
\text { d’interprétation auprès de la clientèle }\end{array}$ \\
\hline Bénéfices socioéconomiques & $\begin{array}{l}\text { - Création d'emplois locaux } \\
\text { - Fournisseurs, partenaires et sous-contractants locaux }\end{array}$ \\
\hline Viabilité économique & $\begin{array}{l}\text { - Rentabilité économique } \\
\text { - Connaissance du marché touristique } \\
\text { - Contribution à la conservation des écosystèmes }\end{array}$ \\
\hline$s$ & \\
\hline
\end{tabular}

Source : Données des auteurs.

\section{Critère 2: \\ Produit touristique de qualité}

L'organisation qui désire offrir un tourisme de nature conforme au label « durable » devrait se doter de plans, de politiques et de programmes de durabilité afin d'implanter le développement durable dans l'ensemble de ses 
sphères de gestion et d'activités et, par le fait même, répondre aux exigences de qualité implicites à la notion de tourisme durable. En ce sens, les clients consommant un produit de tourisme durable devraient s'attendre à voir leurs attentes comblées et à expérimenter un produit de qualité (Tourisme Québec, 2003).

Aussi, tel que souligné dans la Charte du tourisme durable de 1995 (CMTD, 2005), le degré de satisfaction du client quant au produit consommé est-il un critère d'importance dans les efforts de certification du tourisme durable. L'organisation touristique devrait donc s'assurer que son produit répond aux exigences de qualité particulières au secteur d'activités dans lequel elle œuvre et que ses infrastructures d'hébergement rencontrent les exigences de qualité reconnues par les organismes de classification concernés, comme la Fédération des pourvoiries du Québec (FPQ) dans le cas des pourvoiries à droits exclusifs.

Comme la sécurité constitue un des facteurs fortement prisés par la clientèle touristique en matière d'appréciation de l'expérience et contribue même à donner de la crédibilité à l'organisation (CCT, 2004), cette dernière devrait mettre en place les dispositifs nécessaires afin de fournir un produit sécuritaire, autant pour ses clients que pour son personnel (Couture, 2002). Elle devrait veiller à ce que ses activités soient organisées et pratiquées de façon sécuritaire et que ses infrastructures soient entretenues et maintenues constamment en bon état.

\section{Critère 3 : Harmonisation des usages du territoire}

Comme en forêt publique aménagée pour la matière ligneuse, l'utilisation d'un produit a un effet sur les autres produits (Stevens et Montgomery, 2002), l'harmonisation entre les différents exploitants du territoire, notamment les industries touristique et forestière, devrait se faire de façon à prendre en compte les intérêts de chacun. II est reconnu que les activités des industries forestières peuvent avoir un impact majeur sur la qualité visuelle des paysages (Pâquet et Bélanger, 1999), de même que sur la tranquillité des sites de récréation, en raison, par exemple, du bruit des machineries. Or, l'assurance du maintien de paysages de qualité, ou esthétiquement acceptables, et de la tranquillité d'un site de tourisme de nature représente un élément essentiel à l'obtention du label «durable». Le gestionnaire touristique devrait alors déployer les moyens nécessaires pour établir des ententes d'harmonisation avec l'industrie forestière présente sur son territoire, de façon à ce que les opérations de cette dernière n'altèrent pas de façon significative la qualité du milieu naturel. Elle pourrait établir, par exemple, des ententes sur les modalités spatiales et temporelles de leurs activités respectives.

Aussi, en plus d'assurer la sécurité liée à son offre touristique, l'organisation devrait-elle s'assurer que l'accès au site et le réseau routier qui le parcourt sont sécuritaires. À cet effet, elle devrait négocier des ententes avec l'exploitant forestier qui partage son territoire dans le but d'établir des mesures de sécurité routière pour toute personne empruntant les mêmes chemins que ceux utilisés pour le transport du bois.

\section{Critère 4 : \\ Conservation des écosystèmes}

Le tourisme de nature durable se doit de conserver les écosystèmes et de contribuer à la sauvegarde des habitats et des collectivités fauniques et floristiques (OMT, 2004). Si le tourisme de nature, qui base ses activités directement sur les ressources naturelles, n'est pas géré de manière durable, cela peut mener à la détérioration de l'environnement et mettre en péril sa propre viabilité.

L'organisation touristique devrait donc adopter des pratiques durables pour conserver le patrimoine naturel et minimiser les impacts négatifs de ses propres infrastructures et activités sur les espèces sauvages et les habitats fauniques (OMT, 2004). Par exemple, comme la Charte européenne du tourisme durable dans les espaces protégés (FPNRF, 1998) le préconise, elle devrait gérer les flux touristiques de manière à ce que le nombre de visiteurs n'excède pas la capacité de charge du milieu. Aussi, en adoptant un mode de gestion durable de ses activités et de ses infrastructures, l'organisation touristique devrait-elle limiter la pression sur les habitats et les zones naturelles sensibles et minimiser l'altération de la dynamique des populations animales. Elle devrait, par exemple, éviter toute action pouvant engendrer un changement dans les habitudes de migration des espèces, une augmentation de la prédation, une fragmentation du territoire, une altération abusive du couvert végétal ou tout autre phénomène susceptible de mettre en péril l'authenticité écologique.
Elle devrait aussi faire un suivi et des études d'impacts afin d'évaluer les pressions et les menaces présentes ou potentielles exercées par ses infrastructures et la pratique de ses activités touristiques sur le milieu.

Comme la qualité de l'eau et des sols sont des éléments essentiels au fonctionnement et donc à la conservation des écosystèmes, l'organisation devrait mettre en œuvre les moyens nécessaires afin d'y minimiser les impacts négatifs. Elle devrait assurer le maintien d'une eau potable et la conservation des milieux et des habitats aquatiques nécessaires à la survie de plusieurs espèces fauniques et floristiques. De plus, elle devrait s'assurer du maintien de la qualité des sols, élément essentiel à la conservation du substrat de la végétation forestière (CCMF, 2003), en évitant, par exemple, la minéralisation des sols et l'érosion des sentiers.

Pour pouvoir se conformer aux législations, notamment à la Loi sur la conservation et la mise en valeur de la faune, l'organisation touristique devrait posséder une connaissance des espèces menacées et vulnérables, de même que des habitats et des écosystèmes à haute valeur de conservation présents sur son territoire. L'inventaire et la localisation de ceux-ci, réalisés par l'organisation elle-même ou par la consultation des renseignements fournis par les ministères concernés, permettront ainsi de faire une planification plus durable des usages du milieu.

Étant donné la popularité croissante des activités liées à l'observation de la nature (Couture, 2002), l'organisation touristique devrait identifier les enjeux environnementaux qui y sont associés, de même que les législations et les réglementations afférentes. Elle devrait ensuite œuvrer de façon à limiter le dérangement des populations animales en évitant, par exemple, d'appâter les animaux comme cela se fait parfois en forêt pour l'observation de l'ours noir.

Dans le cas d'une organisation touristique qui propose des activités de prélèvement faunique comme la chasse, la pêche et le piégeage, une évaluation du territoire utilisé devrait être effectuée afin de connaître les possibilités d'offre, le niveau d'exploitation optimal et les modalités de gestion durable relatifs à de telles activités. L'organisation devrait donc évaluer et faire un suivi du potentiel faunique des espèces faisant l'objet de prélèvements récréatifs, de la demande exprimée en termes 


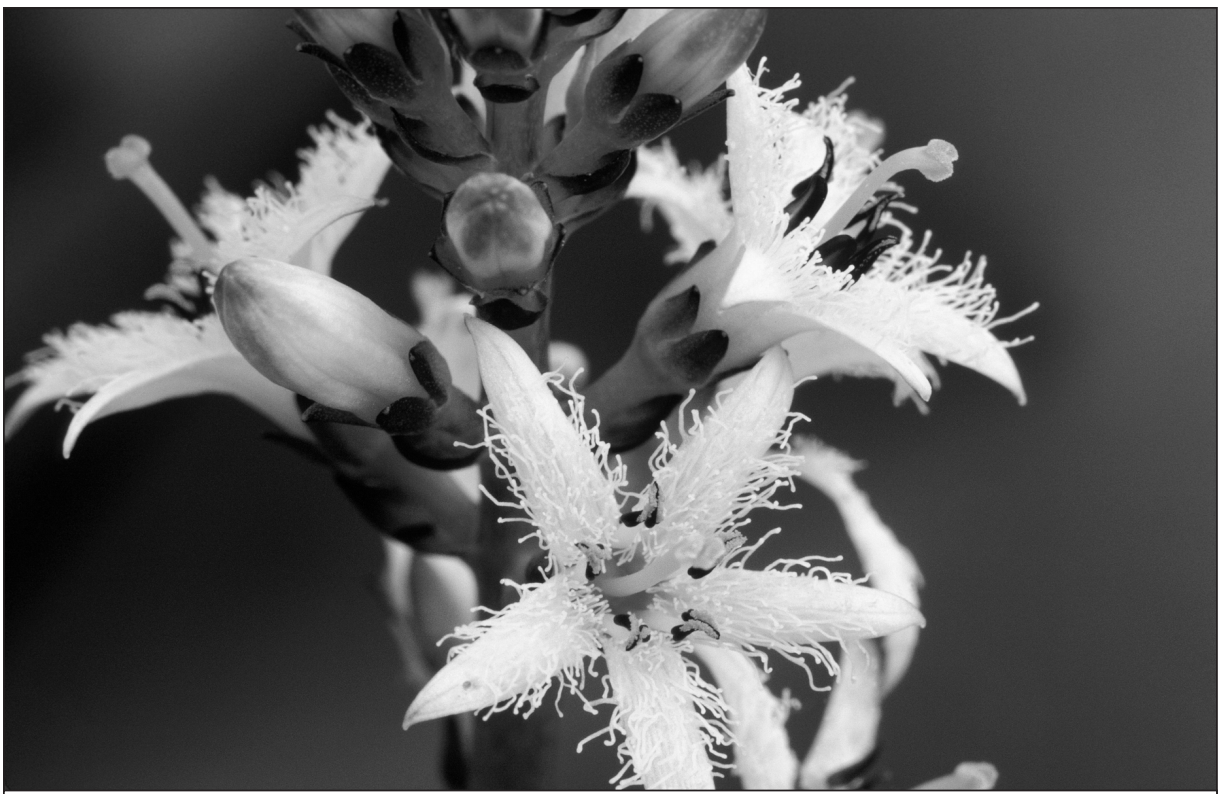

Trèfle d'eau sur l'Île-d'Anticosti, région touristique de Duplessis (Québec). Photo : Jose Schell/Tourisme Québec

de fréquentation des pôles d'attraction du site, des contraintes, de même que de la productivité nette des espèces, afin d'obtenir la densité cible de chacune d'entre elles (MLCP, 1991). Elle devrait en fait s'assurer de faire un prélèvement durable à rendement soutenu en maintenant les potentiels fauniques et en appliquant les réglementations issues de la Loi sur la conservation et la mise en valeur de la faune pour la chasse et le piégeage et de la Loi sur les pêches pour la pêche.

\section{Critère 5 : Gestion de la pollution}

L'organisation touristique voulant offrir du tourisme de nature dit « durable » devrait œuvrer de façon à minimiser la pollution et le gaspillage de toutes sortes, de même que la consommation non durable (WWF, 2001) et ce, dans chaque sphère de gestion de son site. En faisant une utilisation efficace des ressources et de l'énergie, elle réduira ses frais de fonctionnement, tout en contribuant à la protection des ressources renouvelables et non renouvelables.

Tout d'abord, l'organisation touristique devrait faire une gestion durable de ses matières résiduelles en minimisant ses émissions de déchets et en mettant en place les programmes et les infrastructures nécessaires au recyclage et au compostage de toutes les matières recyclables et organiques. Elle devrait aussi mettre en place les mesures nécessaires à la réduction des impacts négatifs de ses eaux résiduelles sur la qualité de tout type de point d'eau environnant et se conformer aux normes municipales et provinciales (MDDEP, 2005) en matière d'eaux usées, d'eau de surface, d'eaux de récréation, d'eaux souterraines, etc. Elle devrait aussi se doter de dispositifs efficaces de gestion de la consommation d'eau (FPNRF, 1998) afin d'éviter le gaspillage d'eau potable.

De plus, l'organisation devrait diminuer sa consommation d'énergie en favorisant les systèmes d'économie d'énergie (interrupteurs de présence, éteinte automatique des lumières, etc.) ; elle devrait par ailleurs tendre davantage vers l'utilisation de sources d'énergie alternatives et/ou renouvelables (énergie solaire, éolienne, etc.) (WWF, 2001). Elle devrait adopter des modèles de consommation durables (CMTD, 1995) et devrait, en plus de réduire sa consommation de matériel, s'approvisionner au maximum en produits biodégradables, recyclables, recyclés, non dommageables pour l'environnement et équitables pour les sociétés.

Enfin, l'organisation touristique devrait éviter le plus possible l'utilisation de véhicules à moteur (WWF, 2001) pour l'entretien de ses infrastructures et pour les déplacements offerts aux visiteurs qui désirent se rendre aux sites d'intérêt sur son territoire. Elle devrait alors favoriser des moyens de transport non motorisés ou, à tout le moins, des véhicules moins dommageables pour l'environnement, comme ceux munis de moteur à quatre temps (Tourisme Québec, 2003).

\section{Critère $6:$ Conservation et mise en valeur du patrimoine culturel et historique}

En forêt publique québécoise, les attributs culturels et historiques sont nombreux et diversifiés (culture autochtone, patrimoine culturel bâti, histoire de la découverte du territoire, culture francophone, etc.). L'organisation de tourisme de nature qui se veut durable devrait posséder une connaissance approfondie du patrimoine culturel et historique du territoire sur lequel elle œuvre afin de le protéger, de le mettre en valeur et d'y minimiser ses impacts négatifs. Elle devrait donc assurer la conservation des artefacts et des sites d'intérêt culturel présents sur son site, mais aussi éviter l'uniformisation des paysages bâtis en s'assurant que les nouvelles infrastructures sont esthétiques, harmonieusement intégrées aux paysages et que la restauration du patrimoine bâti est fidèle à l'histoire et à la culture (WWF, 2001).

Selon la Charte du tourisme durable (CMDT, 1995), I'organisation devrait aussi limiter l'envahissement des communautés locales par les visiteurs afin d'éviter la transformation de leur spécificité culturelle et de leur dynamique traditionnelle, ce qui s'applique principalement aux communautés autochtones en forêts publiques québécoises. Elle devrait promouvoir un respect interculturel (OMT, 2004) des touristes envers les populations visitées de façon à conserver les richesses culturelles de ces dernières, tel que le préconise Tourisme Québec (2003) dans son principe directeur 4.2 du plan d'action 20032008 ( Valorisation du patrimoine culturel et des cultures locales »). En conservant les valeurs traditionnelles et l'authenticité socioculturelle des communautés d'accueil locales et autochtones, un des éléments à la base même de l'attrait touristique des régions québécoises est en conséquence conservé (Tourisme Québec, 2003).

Dans un même ordre d'idées, le tourisme durable doit respecter l'authenticité socioculturelle des communautés hôtes (OMT, 2004). Cela signifie que l'organisation touristique désirant offrir des activités d'interprétation des cultures devrait s'assurer que les éléments et les événements (costumes, danses, légendes, etc.) employés reflètent l'authenticité de ces cultures.

Comme dans tout secteur économique, l'organisation de tourisme de nature durable se doit de respecter les droits ances- 
traux et issus de traités des collectivités locales, notamment des communautés autochtones (Ville de Québec, 2002). Qu'il s'agisse de droits concernant les ressources renouvelables, comme la chasse, la pêche, la trappe, etc., ou tout autre droit territorial (CCMF, 2003), elle ne doit pas agir de façon à les brimer.

\section{Critère 7 : \\ Concertation, sensibilisation et information sociales}

Un des principes fondamentaux dans l'application du tourisme durable est de favoriser la participation des clientèles touristiques, des communautés locales et de tous les intervenants concernés et d'accroître leurs connaissances en matière de durabilité, d'environnement et de culture locale (WWF, 2001). Grâce à l'éducation, aux consultations et aux partenariats, ils sont tous plus susceptibles de développer un sentiment d'appartenance face au projet touristique et sont davantage sensibilisés et impliqués dans la lutte contre les pressions touristiques sur le patrimoine naturel et culturel. L'organisation touristique devrait donc favoriser la participation du public, notamment des collectivités locales, dans son processus décisionnel et ses actions (CMTD, 1995), assurer un processus d'information témoignant d'un souci de transparence et prendre en compte les considérations des divers re- présentants du public (groupes de conservation, industriels forestiers, autochtones, etc.) (OMT, 2004) afin d'intégrer l'ensemble des valeurs sociales.

Aussi, pour pouvoir appliquer concrètement les principes de durabilité à tous ses champs d'activité, l'organisation devrait-elle s'assurer que ses employés possèdent les connaissances et les compétences nécessaires en la matière. Elle devrait non seulement « générer parmi sa direction et son personnel une prise de conscience sur les questions environnementales et culturelles locales, nationales et globales" (Ville de Québec, 2002), mais aussi les former quant aux notions de tourisme durable, d'éthique, d'économie des ressources et d'énergie (FPNRF, 1998). De plus, l'organisation touristique offrant des activités guidées, par exemple des activités de plein air ou d'interprétation, devrait veiller à ce que ses guides possèdent les compétences nécessaires et une formation appropriée (Tourisme Québec, 2002) leur permettant d'offrir un produit sécuritaire et de qualité.

Bien que par définition l'offre d'activités d'interprétation ne soit pas impérative en tourisme de nature durable comme elle l'est en écotourisme, le volet information et éducation revêt un intérêt tel qu'il justifie sa présence dans tout type de tourisme durable (WWF, 2001). Comme ce volet joue un rôle important dans le principe de sensibilisation

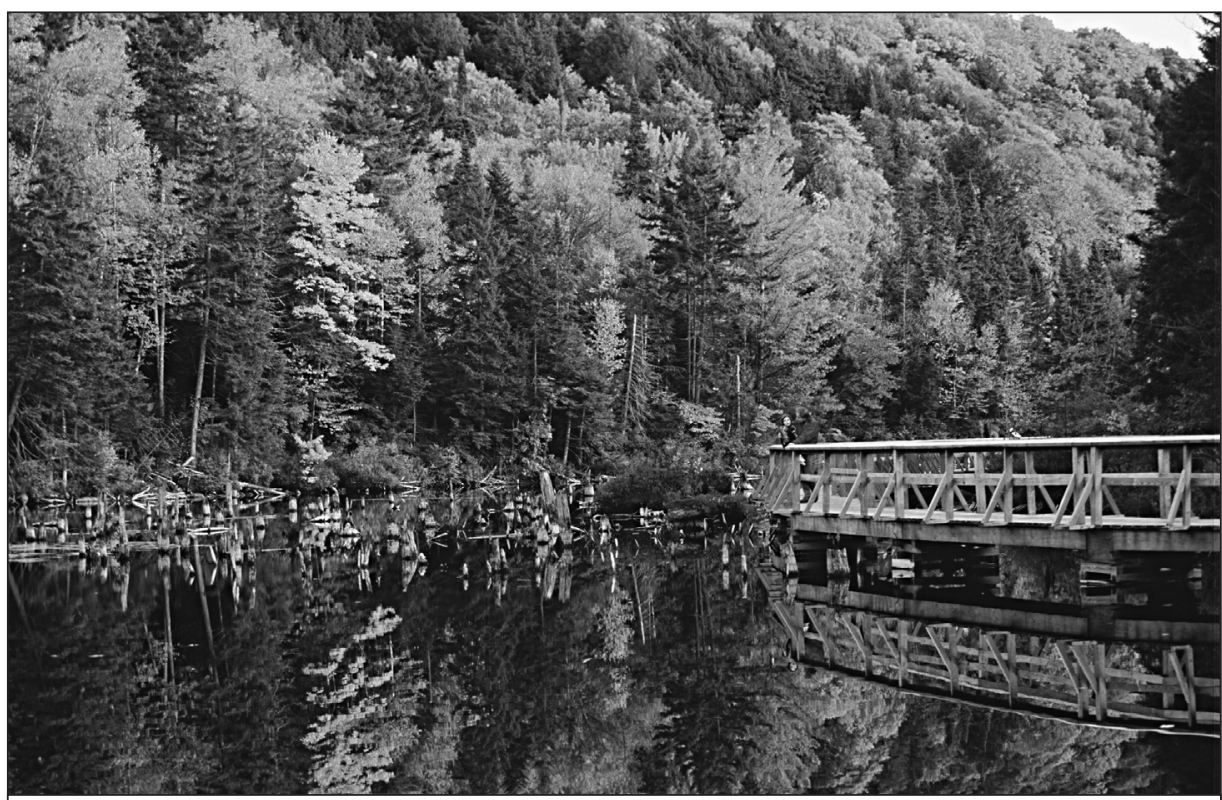

Écotourisme dans la région de Sainte-Agathe-des-Monts (Québec).

Photo: Heiko Wittenborn/Tourisme Québec du public aux valeurs environnementales et sociales de durabilité, il paraît essentiel que l'organisation inclue un minimum d'interprétation et de contenu pédagogique dans son offre de tourisme de nature (FPNRF, 1998), que ce soit comme élément principal ou secondaire de l'activité touristique offerte. L'organisation devrait donc se doter d'un ou de plusieurs programmes et/ou activités d'éducation et d'interprétation, guidés ou autonomes, sur les caractéristiques naturelles et culturelles du milieu, afin de parfaire les connaissances de ses clients en la matière.

Aussi est-il important de conscientiser les clientèles à l'impératif de conserver le milieu, de même qu'aux impacts négatifs que pourraient engendrer leurs actions sur ce dernier (MWF, 2001). Selon la Charte européenne du tourisme durable dans les espaces protégés (FPNRF, 1998), le marketing et la promotion responsables constituent des principes de durabilité essentiels. L'organisation devrait alors mettre sur pied, à l'intention des visiteurs, des programmes d'information sur les valeurs du tourisme durable, les éléments écologiques et culturels sensibles du milieu et les comportements à adopter afin de conserver ces derniers.

\section{Critère 8 : \\ Bénéfices socioéconomiques}

Le tourisme de nature durable est considéré comme un agent de développement économique pour la région hôte et un agent sensible aux besoins socioéconomiques des communautés locales. Puisqu'il doit contribuer à l'amélioration de la qualité de vie de ces dernières (CMTD, 1995), l'organisation désirant offrir du tourisme durable devrait se baser, dans ses différentes sphères d'activités, sur la gamme des possibilités qu'offre l'économie locale et, ainsi, faire une redistribution équitable des revenus d'activités touristiques aux populations locales (OMT, 2004). II s'agit de générer des retombées économiques positives en recourant "de plus en plus, dans ses activités, aux matériaux et aux produits locaux ainsi qu'aux ressources logistiques et humaines locales " (Ville de Québec, 2002). Par exemple, l'organisation devrait assurer un approvisionnement local en produits et services, assurer la création d'emplois locaux (WWF, 2001) en donnant priorité à la main-d'œuvre locale (FPNRF, 1998) et mettre en valeur les produits locaux. 


\section{Critère 9 : Viabilité économique}

La rentabilité et la viabilité sont des fondements du tourisme durable (Tourisme Québec, 2003). L'organisation de tourisme de nature durable devrait conséquemment être rentable et viable (CMTD, 1995) afin de répondre aux impératifs économiques du développement durable, c'est-à-dire participer à l'économie locale et contribuer au développement et à l'amélioration des conditions de vie des populations locales. L'organisation devrait donc s'assurer que son produit est conçu, commercialisé et vendu de façon à être viable à long terme (OMT, 2004).

Comme le démontre l'importante diversification des clientèles qui fréquentent désormais les territoires fauniques structurés, la caractérisation et les exigences des différentes clientèles de tourisme de nature sont en constante évolution. Pour pouvoir s'adapter et répondre de façon efficace aux variations de la demande touristique et tendre, par le fait même, vers une adéquation entre son produit et les attentes réelles des clients, l'organisation devrait adopter une approche marketing et déployer les moyens nécessaires lui permettant d'être à l'affût des tendances du marché et des besoins de ses clients (FPNRF, 1998).

Enfin, en ce qui a trait au tourisme de nature en forêt, le pôle économique du développement durable va jusqu'à prescrire à l'organisation touristique de contribuer à la conservation de l'environnement (WWF, 2001). Ainsi, en réinjectant une partie de ses bénéfices dans des programmes et des actions visant la conservation des écosystèmes forestiers présents sur son territoire, elle contribue à assurer la pérennité des ressources naturelles desquelles dépendent sa propre survie et sa viabilité.

\section{Discussion}

En forêts publiques québécoises, une stratégie d'aménagement intégrée des ressources reste encore à développer, mais surtout à implanter. Si, par celle-ci, l'industrie forestière se doit d'appliquer un aménagement forestier durable, l'industrie touristique devrait, elle aussi, se doter d'une politique de développement durable. Devant l'importance d'assurer la pérennité des ressources, il semble alors légitime de demander à l'ensemble des gestionnaires $œ u v r a n t$ en territoires publics de faire une utilisation durable de celles-ci. Le prochain défi dans l'aménagement des forêts publiques du Québec est donc de doter les territoires à usages multiples, tels les territoires fauniques structurés, d'une politique de tourisme de nature durable acceptée par les ministères concernés. Pour ce faire, ces derniers devront toutefois reconnaître l'importance d'une harmonisation égale des usages et ainsi mettre en œuvre les moyens nécessaires afin que soient respectées les ententes établies entre les industries forestière et touristique dans les plans d'affectation du territoire public (PATP), comme l'ont fait nos voisins de l'Ontario avec leur protocole d'entente d'intendance des ressources (EIR) (Ministère des Richesses naturelles, 2000). Le cadre d'évaluation du tourisme de nature durable que nous avons élaboré et présenté dans cet article est un concept qu'il reste à entériner et qui, nous l'espérons, servira de base de réflexion pour une éventuelle certification à la fois reconnue par Tourisme Québec et implantée dans les forêts publiques québécoises par un organisme de certification.

Véronique Desmarais est adjointe à la coordination pour Partenariat innovation forêt.

Louis Bélanger est professeur à l'Université Laval, Faculté de foresterie et de géomatique.

\section{Bibliographie}

Bélanger, Louis (1998), Mémoire pour l'Union québécoise pour la conservation de la nature, Québec, UQCN, [www.naturequebec.org/ pages/accueil.asp].

Commission canadienne du tourisme (CCT) (2004), A Canadian Study of Indicators Relating to Sustainable Tourism and Ecotourism: The Case Study of Northern Cape Breton, Canada, Parcs Canada.

Conférence mondiale du tourisme durable (CMTD) (1995), Charte du tourisme durable, Espagne.

Conseil canadien des ministres des forêts (CCMF) (2003), Définir l'aménagement forestier durable au Canada, Critères et indicateurs 2003, Canada, $27 \mathrm{p}$.

Couture, Maurice (2002), Écotourisme et espaces naturels, Québec, Université du Québec à Montréal, École des sciences de la gestion, $129 \mathrm{p}$.
Fédération des parcs naturels régionaux de France (FPNRF) (1998), Charte européenne du tourisme durable dans les espaces protégés, France, $26 \mathrm{p}$. Disponible [http://www.ceven nes-ecotourisme.com/modules/wfdownloads/ uploads/charte_europeenne_tourisme_dura ble.pdf].

Lück, Michael, et Torsten Kirstges (2003), Global Ecotourism Policies and Case Studies: Perspectives and Constraints, UK, Channel Press Publications.

Ministère des Ressources naturelles de la faune et des parcs (MRNFP) (2004), La faune et la nature Ça compte!, Gouvernement du Québec, [www.faunenatureenchiffres.gouv.qc.ca].

Ministère des Ressources naturelles et de la faune (MRNF) (2006), Gouvernement du Québec, [www.mrnf.gouv.qc.ca].

Ministère des Richesses naturelles (MNR) (2000), Protocole d'entente entre les industries du tourisme et de la forêt, Gouvernement de l'Ontario, [www.mnr.gov.on.ca/mrn/forests/adr\% 20program/MOU.pdf].

Ministère du Loisir, de la Chasse et de la Pêche (MLCP) (1991), Le cadre de gestion des réserves fauniques, Québec, Gouvernement du Québec.

Ministère du Développement durable, de l'Environnement et des Parcs (MDDEP) (2005), Gouvernement du Québec, [www.mddep.gouv.qc.ca].

Organisation mondiale du tourisme (2002), Déclaration de Québec sur l'écotourisme, Québec, 9 p., [http://www.uneptie.org/pc/tourism/ documents/ecotourism/WESoutcomes/Que bec-Declar-fr.pdf].

Organisation mondiale du tourisme (OMT) (2004), Nouvelle définition du tourisme durable, [www.world-tourism.org], Bulletin électronique $\mathrm{n}^{\circ} 7$, novembre, Espagne.

Pâquet, Josée, et Louis Bélanger (1999), «Qualité des paysages forestiers, une harmonisation difficile mais possible ", Canada, Naturaliste Canadien, hiver, p. 29-35.

Stevens, J.A., et C.A. Montgomery (2002), A Synthesis of Multi-resource Research with Application to the Pacific Northwest: Multiple Use, Tradeoffs, and Joint Production, ÉtatsUnis, US Department of Agriculture, Forest Service, Pacific Northwest Research Station.

Tourisme Québec (2003), Nature et tourisme au Québec, Orientations et plan d'action 2003-2008, Québec, Gouvernement du Québec.

Weaver, David B. (2001), The Encyclopaedia of Ecotourism, New York, Cabi Publishing.

World Travel and Tourism Council (WTTC) (2004), Certification Green Globe, Australie, [http:// www.greenglobe21.com/History.aspx].

World Wildlife Fund (WWF) (2001), The WWF mission and tourism, UK, WWF. 